\section{Groundwork on the troposphere}

\author{
Richard P. Wayne
}

Atmospheric Chemistry: Fundamentals and Experimental Techniques. By Barbara J. Finlayson-Pitts and James N. Pitts, Jr. Wiley: 1986. Pp. 1,098. £57.45, $\$ 59.95$.

Twenty-five years ago Philip Leighton's seminal monograph The Photochemistry of Air Pollution was published. Leighton applied basic chemical kinetic, photochemical and spectroscopic concepts to problems of tropospheric air pollution. A striking development of the 1950 s had been the recognition that photochemical reactions initiated by sunlight could convert relatively innocuous primary pollutants into substances that created health hazards, nuisance and economic loss. One of Leighton's outstanding contributions was in bringing together the available knowledge about chemical processes and absorption rates in the atmosphere in an attempt to describe the conversion of the initial pollutants to final products, the underlying motivation being not only the scientific interest of the problem but also the possible applications to the control of pollution.

Since 1961, an enormous amount of research has been conducted in the laboratory on chemical transformations, and in the field on sources and concentrations of pollutants. Computer models have made possible realistic chemical bases for pollution control strategies. Jim Pitts and Barbara Finlayson-Pitts have been major contributors to much of this research. Their new book is a worthy successor to Leighton's, adopting a similar approach that is strongly grounded in laboratory chemical experiments.

The atmospheric chemistry treated is concerned mainly with the Earth's troposphere, although the impact of tropospheric chemical processes on stratospheric ozone is considered briefly. The thrust of the book is clearly directed towards the sources, chemistry and control of air pollution. The particular topics discussed include photochemical smog, acid deposition, toxic volatile organic compounds, airborne mutagenic polycyclic aromatic hydrocarbons, and primary and secondary particles in the atmosphere. However, the authors show that there is a close interrelation between these various manifestations of pollution, the photochemical and thermal reactions of a relatively small number of species making up the overall complex chemistry and physics. In many cases, the "polluted" troposphere shows in exaggerated form the chemistry of the natural troposphere. For example, the ozone and the oxides of nitrogen that are features of photochemical smog are important species in the troposphere remote from man's activities and air pollutant emissions, and sulphur compounds and hydrocarbons are released to the atmosphere by natural biogenic processes. The authors therefore review the chemistry of the natural troposphere as a special topic.

Roughly half of the book is devoted to presenting the principles of kinetics, spectroscopy and photochemistry that are applicable to atmospheric chemistry, and to the kinetics and mechanisms of important specific gas phase reactions in real and simulated atmospheres. Other important parts of the book deal with modelling studies, and with the physics and chemistry of aerosols and the mechanisms of their formation. I particularly appreciated the descriptions throughout the book of how experiments are really performed. For example, there is a comprehensive discussion of flow, flash, modulation and static

\section{Myths of Mars}

\section{James Trefil}

To Utopia And Back: The Search for Life in the Solar System. By Norman H. Horowitz. W.H. Freeman: 1986. Pp.168. Hbk $\$ 17.95, £ 17.95 ;$ pbk $\$ 11.95, £ 11.95$.

"THE idea of an inhabited Mars occupies a special place in our culture." With this comment, Norman Horowitz touches off a short but fascinating discussion of the process by which our modern view of Mars was forged. As the former head of the bioscience section of the Mariner and Viking missions, he had a ringside seat during that exciting period in the 1970 s when the human race, for the first time, sent probes to the surface of our sister planet.

This is not, however, a dry recitation of technical achievements, although the setting up of a chemistry laboratory capable of operating and sending back useful information over interplanetary distances is surely one of the great technical feats of all time. Instead, Horowitz takes us on a tour of the development of our ideas about the origin of life, from spontaneous generation to chemical evolution, showing at each step how misconceptions about life tricked people into believing that all the planets of the Solar System were populated by living beings. He deals with and sets the record straight about things such as the canals of Mars and Percival Lowell's widely read views on Mars as an abode of life. He then carries this discussion forward into the $1960 \mathrm{~s}$, when a prestigious panel assembled by NASA stated that the Martian atmosphere was similar to our techniques for determining rate constants in the gas phase, in solution and on solid surfaces, together with descriptions of methods for the generation and detection of reactive reaction intermediates. Similarly valuable accounts are given of techniques for monitoring pollutants, the use of environmental ("smog") chambers and the analysis of particles.

By any standards this book is a major work. It is authoritative and comprehensive. The text abounds with tables and figures of factual data to back up the themes presented, and the references provide an overview of research published up to mid-1985. Atmospheric Chemistry will long remain a valuable text and reference book describing the chemistry of the gases, droplets and particles present in the natural and polluted troposphere.

Richard $P$. Wayne is University Lecturer in Physical Chemistry, Physical Chemistry Laboratory, University of Oxford, South Parks Road, Oxford $O X 13 Q Z, U K$, and Dr Lee's Reader in Chemistry at Christ Church, Oxford.

own. It seems that whenever we have had a chance, we have interpreted our data in such a way as to produce a picture of an Earth-like Mars.

In the modern debate over the existence of extraterrestrial intelligence, this proclivity has been elevated to something called the "Principle of Mediocrity", which states that the Earth does not occupy a special place in the cosmos. This principle is accepted without proof - indeed, for some writers it has taken on the mantle of dogma. Yet, as Horowitz shows, when it is applied to the one body of which we have extensive knowledge, it fails miserably. In a masterly summary of the search for life on Mars by the Viking landers, he discusses each of the experiments carried out and shows how they establish that Mars, with its highly reducing environment, is far more hostile to life than we had ever imagined.

Of course, a few diehards refuse to give up the ancient myth of an inhabited Mars. Horowitz dismisses their theories, rightly I think, as "daydreams", and variations of the "blue unicorn theory".

All in all, this is a well-written, nontechnical book. Anyone who, like myself, has followed Martian exploration at a distance, and, would like to have a clear, concise summary of what we now know, will find it makes excellent reading. I only wish that Horowitz had told us a little more about his own emotions and reactions as this crucial step in mankind's knowledge of the Solar System was made.

James Trefil is a Professor in the Department of Physics, University of Virginia, Charlottesville, Virginia 22901, USA. His latest book is Meditations at 10,000 Feet (Charles Scribner's Sons, 1986). 